\title{
Spatially coherent broadband IR source
}

\author{
Stuart Yin, Paul Ruffin, Christina Brantley, Eugene Edwards, \\ Chia-En Yang, Jimmy Yao, and Claire Luo
}

An extremely broadband IR supercontinuum source (up to 7 microns) can be generated by employing high transmission and high laser damage threshold IR waveguides.

Spatially coherent broadband IR sources are used for many applications, such as obtaining the spectroscopic signatures of remote targets in real time and IR optical coherence tomography (OCT). Because most biomaterials have IR spectroscopic signatures, IR OCT can provide information about the 3D shapes and the chemical composition of biomedical samples. Although IR lasers such as quantum cascade lasers (QCLs) are spatially coherent sources, they have very narrow spectra. Widely tunable IR lasers that can cover the entire near- to mid-IR spectral range are not yet available. However, we have found that an IR supercontinuum (SC) source can be an excellent candidate for spatially coherent broadband IR applications.

SC generation (SCG) is a process where short laser pulses are launched into optical fibers and waveguides to create a spatially coherent broadband source. ${ }^{1}$ As a result of the nonlinear interactions between the light signal and the nonlinear optical medium (including self-phase modulation, self-steepening, and delayed Raman response), the narrow spectrum of the input laser pulse is largely broadened. Although SCG has been very successful in the visible and the near-IR spectral regime, it is very challenging to successfully use high-power, high-energy SCG in the midIR and far-IR regime due to the lack of high transmission rates and the need for a high laser damage threshold in IR fibers and waveguides.

In our research, we worked with single-crystal sapphire fiber because it has good IR transmittance up to 3.1 microns (the linear absorption is only $0.5 \mathrm{~dB} / \mathrm{m}$ at 2.94 microns), a high laser damage threshold because of the very high melting temperature (over $2000^{\circ} \mathrm{C}$ ), and good thermal conductivity $\left(36 \mathrm{~W} / \mathrm{m}^{\circ} \mathrm{C}\right) .^{2} \mathrm{Re}-$ cently, we demonstrated broadband (visible to mid-IR range) SC performance in single-crystal sapphire fibers (see Figure 1). ${ }^{3-5}$
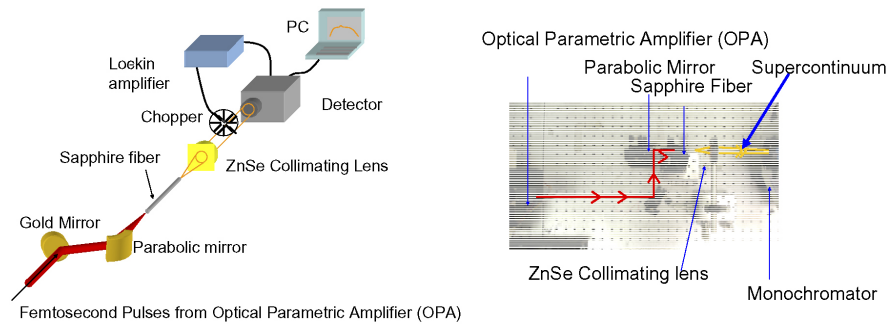

Figure 1. Sketch (left) and picture (right) of the IR supercontinuum generation (SCG) setup. ZnSe: Zinc selenide.

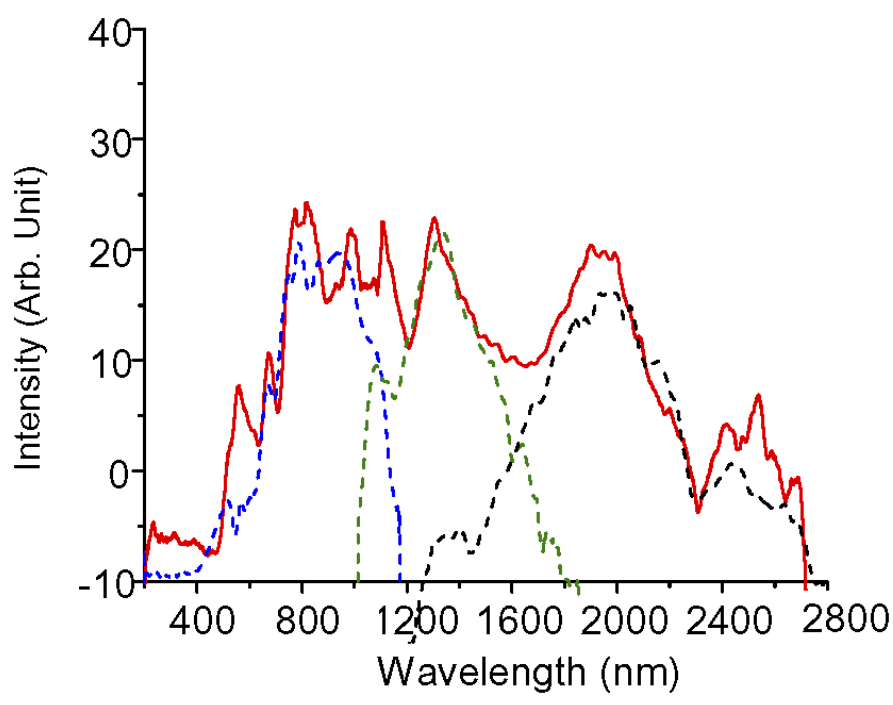

Figure 2. Experimentally measured SCG with simultaneous pumping at three wavelengths in single-crystal sapphire fiber (blue dash: $784 \mathrm{~nm}$, green dash: 1290nm, black dash: 2000nm, red solid: the three pumping sources together). ${ }^{5}$

Experimentally measured SC output for this single-crystal sapphire fiber from three pumping wavelengths is shown in Figure 2.

To further extend the range in the mid-IR spectral regime, we also investigated other unconventional IR fibers and waveguides. 


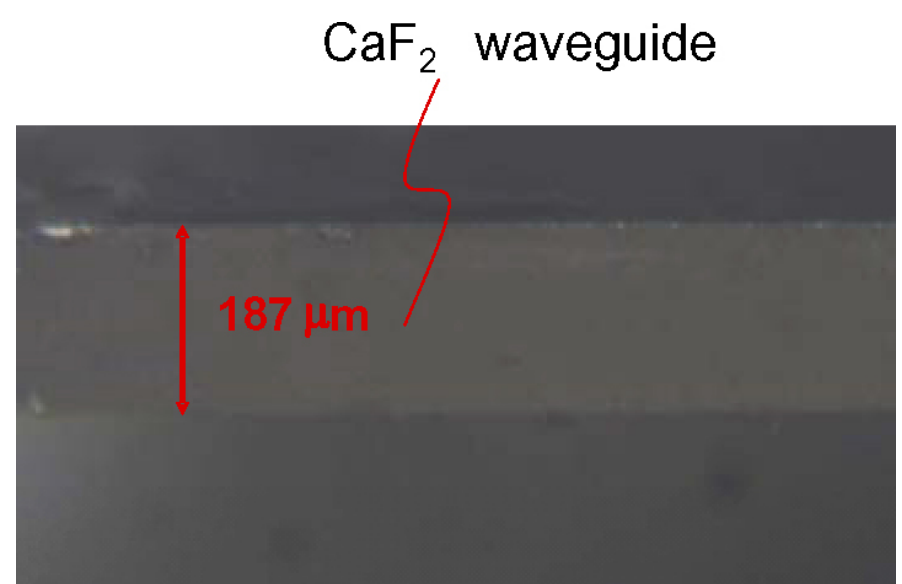

Figure 3. A fabricated one-dimensional calcium fluoride $\left(\mathrm{CaF}_{2}\right)$ waveguide.

Specifically, we chose calcium fluoride $\left(\mathrm{CaF}_{2}\right)$ because it has a good IR transmittance (up to 8 microns) and a high melting temperature $\left(>1000^{\circ} \mathrm{C}\right) \cdot \mathrm{CaF}_{2}$ waveguides can be fabricated using a precise lapping and polishing approach. Figure 3 shows a fabricated $\mathrm{CaF}_{2}$ waveguide with a thickness of approximately 187 microns and a length of about $2.5 \mathrm{~cm}$.

We quantitatively simulated the performance of SCG in the $\mathrm{CaF}_{2}$ waveguide, assuming that it measured 25 microns in diameter and $5 \mathrm{~cm}$ in length. We also assumed that the pumping light had the following parameters: $150 \mathrm{fs}$ pulse duration, $50 \mathrm{MW}$ maximum peak power, and a 3 micron wavelength. We used finite difference time domain software to simulate the SCG under peak power levels 1, 5, 10, and 50MW (see Figure 4). From this simulation result, we concluded that a very broad SC spectrum, covering the entire 2-7 micron spectral regime, can be realized.

Although the SC at shorter wavelengths ( $<2$ microns) is not shown in Figure 4 due to the memory limitation of the simulation software, we expect there would also be good SCG in the shorter wavelength region based on the tendency of this SC spectral profile. Such an extremely broad spectral profile would be very useful for standoff sensors and imagers because it covers the vibrational and rotational energy levels of many materials.

It is possible to generate an extremely broadband IR SC source (up to 7 microns) by employing high transmission and high laser damage threshold IR waveguides, which can greatly enhance the capabilities of standoff sensors and imagers. It may also enable new applications for OCT, such as determining the chemical composition of biomedical samples. We will continue our experimental investigations of $\mathrm{SC}$ in $\mathrm{CaF}_{2}$ waveguides with these applications in mind.

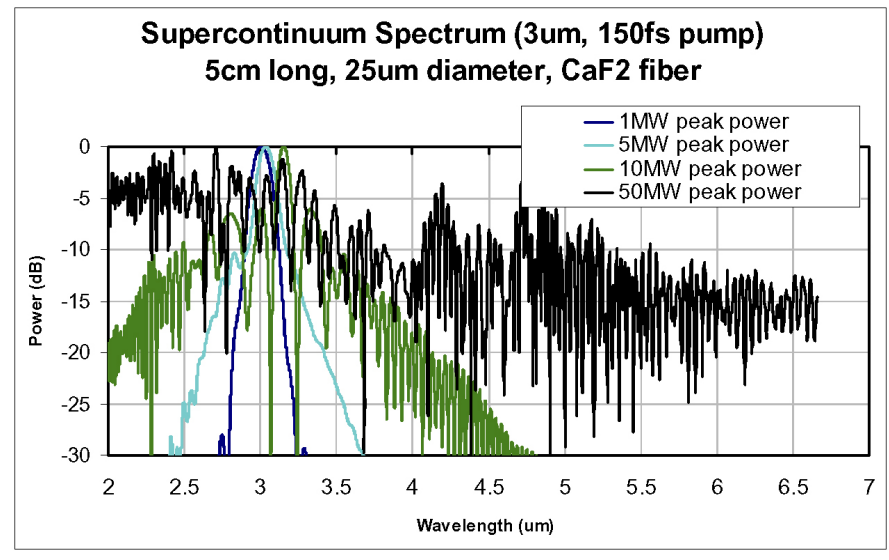

Figure 4. The simulated SCG as a function of pump light peak power, pumped at 3 microns for a 5cm-long, 25 micron-diameter $\mathrm{CaF}_{2}$ waveguide.

The authors acknowledge the partial support of this work by the Office of Naval Research basic research program and an Army Small Business Innovation (SBIR) Research Phase I project.

\section{Author Information}

\section{Stuart Yin, Chia-En Yang, and Jimmy Yao}

Department of Electrical Engineering

The Pennsylvania State University

State College, PA

Stuart (Shizhuo) Yin is a professor at the Pennsylvania State University, where he also received his $\mathrm{PhD}$ in electrical engineering in 1993. He has over 20 years of experience developing advanced optical materials, devices, and techniques for optical sensing, communications, and imaging. He has authored and coauthored over 100 papers in a variety of prestigious optics journals. He has also coauthored three books: Photorefractive Optics (Academic Press, 2000), Introduction to Information Optics (Academic Press, 2001), and Fiber Optic Sensors (CRC Press, 2008). In the past 10 years, he has successfully completed over 10 research projects with total funding close to $\$ 10$ million. For his contribution to the optics field, he was elected a fellow of the International Society for Optical Engineering in 2004 and a Fellow of the Optical Society of America in 2007. He is also the recipient of a 1996 US Army Young Investigator Award and a 2004 Penn State Engineering Society' Outstanding Research Award. 
Chia-En Yang is a PhD candidate.

Jimmy Yao is a PhD candidate.

Paul Ruffin, Christina Brantley, and Eugene Edwards US Army AMRDEC

Huntsville, AL

Paul Ruffin is a senior research scientist.

Christina Brantley is an engineer.

Eugene Edwards is an engineer.

\section{Claire Luo}

General Opto Solutions, LLC

State College, PA

Claire Luo is a senior research and development engineer. She received her master's degree in computer science and engineering from the Pennsylvania State University in 2000. She successfully worked on several optics-related SBIR contracts during and after her graduate study. She has published key papers in a variety of optics areas, including IR SCG, microfabrication by femtosecond lasers, and ultrafast optical beam scanning.
References

1. R. Alfano, The Supercontinuum Laser Source, 2nd ed., Springer, Berlin, 2006.

2. J. Harrington, Infrared Fibers and Their Applications, p. 7, 2004.

3. S. Yin, J. Kim, P. Ruffin, and C. Luo, Supercontinuum generation, Opt. Commun. 281, pp. 1113-1117, 2008.

4. J. Kim, S. Yin, P. Ruffin, E. Edwards, C. Brantley, C. Luo, et al., Broadband IR supercontinuum generation, Opt. Express 16, pp. 4085-4093, 2008.

5. J. Kim, S. Yin, P. Ruffin, E. Edwards, C. Brantley, C. Luo, et al., Broadband supercontinuum by using three pumping sources, Opt. Express 16, p. 14792, 2008. 